\title{
Versorgungsforschung für den Öffentlichen Gesundheitsdienst (ÖGD) und das öffentliche Gesundheitswesen
}

\section{Health Services Research for the Public Health Service (PHS) and the Public Health System}

\author{
Autoren \\ Institut
}

\author{
A. Hollederer, M. Wildner
}

Bayerisches Landesamt für Gesundheit und Lebensmittelsicherheit (LGL), Nürnberg
Schlüsselwörter

- Versorgungsforschung

- öffentlicher Gesundheitsdienst

- Gesundheitswissenschaften

- Gesundheitsberufe

o öffentliches Gesundheitswesen

- Gesundheitsamt

Key words

health services research

- public health services

- science of public health

- health professions

- public health system

- local health office

\section{Bibliografie}

DOI http://dx.doi.org/

10.1055/s-0034-1390410

Online-Publikation: 7.11.2014

Gesundheitswesen 2015;

77: 232-236

(c) Georg Thieme Verlag KG

Stuttgart · New York

ISSN 0941-3790

\section{Korrespondenzadresse}

PD Dr. Alfons Hollederer, MPH

Bayerisches Landesamt für

Gesundheit und Lebensmittelsicherheit (LGL)

Schweinauer Hauptstraße 80

90441 Nürnberg

alfons.hollederer@Igl.bayern.de

\section{License terms}

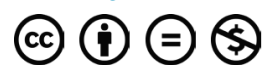

\section{Zusammenfassung}

\section{V}

Ausgangslage: Es besteht ein großer Bedarf an Versorgungsforschung für den Öffentlichen Gesundheitsdienst und das öffentliche Gesundheitswesen in Deutschland. Der Öffentliche Gesundheitsdienst ist innerhalb der Versorgungsforschung aber unterrepräsentiert. Dafür sind strukturelle, historische und fachgebietsbezogene Gründe verantwortlich. Das Tätigkeitsfeld zeichnet sich durch ein breit gefächertes Aufgabenspektrum, entsprechend hohe Qualifizierungsbedarfe und sich verändernde Rahmenbedingungen aus.

Ergebnisse: Das Konzept der Versorgungsforschung bietet mit seinen Prinzipien der Ergebnisorientierung, Multidisziplinarität, Multiprofessionalität und Alltagsorientierung gute Ansatzpunkte für die Adaption im Öffentlichen Gesundheitsdienst und öffentlichen Gesundheitswesen. Im Mittelpunkt des Beitrags steht ein systemtheoretisches Modell speziell für die Versorgungsforschung im Öffentlichen Gesundheitsdienst und öffentlichen Gesundheitswesen. Es baut auf bestehenden Modellen der Versorgungs- und Gesundheitssystemforschung auf und entwickelt sie für die besonderen Anforderungen im Öffentlichen Gesundheitsdienst weiter. Es bildet eine theoretische Grundlage für die Versorgungsforschung auf der Makro-, Mesound Mikroebene im Öffentlichen Gesundheitsdienst und öffentlichen Gesundheitswesen.

Schlussfolgerungen: Zukunftsperspektiven werden für den Öffentlichen Gesundheitsdienst in der fachgebietsbezogenen Weiterentwicklung von „Old Public Health“ zu „New Public Health“ und in der Integration von Versorgungs- und Gesundheitssystemforschung gesehen. Großes Entwicklungspotenzial liegt in der stärkeren Vernetzung von universitärer Forschung und Öffentlichem Gesundheitsdienst wie sie exemplarisch in der „Pettenkofer School of Public Health München" aufgebaut wird.

\section{Abstract \\ $\nabla$}

Introduction: There is a great need for health services research in the public health system and in the German public health service. However, the public health service is underrepresented in health services research in Germany. This has several structural, historical and disciplinary-related reasons. The public health service is characterised by a broad range of activities, high qualification requirements and changing framework conditions.

Results: The concept of health services research is similar to that of the public health service and public health system, because it includes the principles of multidisciplinarity, multiprofessionalism and daily routine orientation. This article focuses on a specified system theory based model of health services research for the public health system and public health service. The model is based on established models of the health services research and health system research, which are further developed according to specific requirements of the public health service. It provides a theoretical foundation for health services research on the macro-, meso- and microlevels in public health service and the public health system.

Discussion: Prospects for public health service are seen in the development from "old public health" to "new public health" as well as in the integration of health services research and health system research. There is a significant potential for development in a better linkage between university research and public health service as is the case for the "Pettenkofer School of Public Health Munich”. 


\section{Einleitung}

\section{$\nabla$}

„Insofern nun die vornehmste Aufgabe des öffentlichen Arztes darin liegt, dass er Ursprung und Hauptursachen verschiedener Krankheiten, denen die Staaten unterworfen sind, in einer strengen Prüfung erforsche, so will ich nur eine einzige von dieser ansehnlichen Versammlung kurz untersuchen, nämlich das Elend des Volkes als die fruchtbarste Mutter der Krankheiten“ begann Johann Peter Frank im Jahr 1790 seine berühmte „Akademische Rede vom Volkselend als der Mutter der Krankheiten“ in Pavia [1]. Johann Peter Frank gilt als der Wegbereiter des öffentlichen Gesundheitswesens und des Öffentlichen Gesundheitsdienstes [2]. Die historischen Ansätze zum Zusammenhang von sozialer Lage und Gesundheit sowie die Rolle des öffentlichen Gesundheitswesens haben in Laufe von mehr als 200 Jahren nichts an Aktualität verloren. Die Forschung zu Determinanten von Gesundheit bildet auch heute die Grundlage für Wirkmodelle und Interventionen für das öffentliche Gesundheitswesen. So hat z. B. vor kurzem die Weltgesundheitsorganisation (WHO) eine eigene Kommission „Social Determinants of Health“ eingesetzt, um in einer weltweiten Strategie zur gesundheitlichen Chancengleichheit die Verbesserung von Gesundheit und Lebenserwartung in einer Generation zu erreichen [3]. Für Europa wurde ein umfassendes WHO-Rahmenkonzept „Gesundheit 2020“ [4] entwickelt. Die WHO formulierte in dieser Strategie „Gesundheit 2020“ [5] einen expliziten Handlungsbedarf für „Überwachung und Evaluierung der Forschung im Bereich der öffentlichen Gesundheit“, da „alle Konzepte und Maßnahmen zur Verbesserung der Gesundheitssituation eine solide Wissensgrundlage voraus(setzen), und zur Umsetzung von ,Gesundheit 2020‘ eine Verbesserung der Evidenzgrundlage im Hinblick auf Maßnahmen im Gesundheitsbereich erforderlich sein (wird)“ [5].

\section{Öffentlicher Gesundheitsdienst und Versorgungsfor- schung: eine Bestandsanalyse \\ $\nabla$}

An dieser Stelle setzt die Versorgungsforschung an, die sich in Deutschland derzeit im Aufbau befindet. Die Versorgungsforschung untersucht systematisch sowohl die Ursachen als auch die Wirkungen von Versorgungsprozessen und -strukturen, um Verbesserungen im Gesundheitssystem zu erreichen [6,7]. Im Fokus stehen in der Regel die Ergebnisqualität und die Wirksamkeit von Konzepten und Maßnahmen. Die Versorgungsforschung umfasst die Deskription, Erklärung, Gestaltung, evaluative Begleitung und die Ergebnisevaluation [8,9]. Zentrale Fragestellungen sind dabei: Welche Ursachen sind verantwortlich? Welche Interventionen lassen sich aufgrund versorgungswissenschaftlicher Ergebnisse entwickeln? Wie ist die Versorgung zu gestalten? Welche Implementations- und Umsetzungsprobleme können auftreten und wie wirksam ist die Intervention unter Alltagsbedingungen? Diese Grundfragen der Versorgungsforschung sind auch für die Weiterentwicklung des öffentliche Gesundheitswesens und des Öffentlichen Gesundheitsdienstes von elementarer Bedeutung.

Das öffentliche Gesundheitswesen definiert sich als der Bereich des Gesundheitswesens, „der von unmittelbaren oder mittelbaren Trägern der Staatsverwaltung oder durch Einrichtungen wahrgenommen wird, die von ihnen errichtet und getragen werden“ [10]. Der Öffentliche Gesundheitsdienst gilt als „3. Säule“ im Gesundheitswesen neben den ambulanten und stationä- ren Sektoren in Deutschland. Er ist der Teil des öffentlichen Gesundheitswesens, der öffentlich-rechtliche Gesundheitsaufgaben einschließlich der Untersuchungstätigkeiten wahrnimmt [10]. Die unterste Ebene im Öffentlichen Gesundheitsdienst bilden die Gesundheitsämter. Die Aufgaben- und Kompetenzbereiche im Öffentlichen Gesundheitsdienst bieten viele Ansatzpunkte und Zugangswege für die Versorgungsforschung. Öffentliche Gesundheit ist „eine anwendungsorientierte Wissenschaft und ein praktisches Handlungsfeld, die sich aus einer Vielzahl von wissenschaftlichen Disziplinen und Professionen speist“ [10]. Laut Ärztestatistik der Bundesärztekammer waren im Jahr 20132432 Ärztinnen und Ärzte allein in den Gesundheitsämtern tätig, von denen 437 die Fachgebietsbezeichnung „Öffentliches Gesundheitswesen“ führten [11]. Neben den Ärzten bilden die Sozialarbeiter die größte Berufsgruppe im Öffentlichen Gesundheitsdienst [12]. $\mathrm{Zu}$ den weiteren Gesundheitsdienstberufen im Öffentlichen Gesundheitsdienst zählen z. B. die nichtärztlichen Psychotherapeuten, Psychologen, Apotheker, Hygienekontrolleure und Gesundheitswissenschaftler (Master of Public Health). Die verschiedenen medizinischen Fachdisziplinen und Berufsgruppen wirken bei der Erbringung der Gesundheitsdienstleistungen im Öffentlichen Gesundheitsdienst zusammen. Die Kernaufgaben sind zusammengefasst (in Anlehnung an [13]):

- Der Gesundheitsschutz (Hygiene, Infektionsschutz, Umweltmedizin, Überwachung im Pharmaziebereich usw.)

- Die Prävention, Beratung, Aufklärung (Schulgesundheitspflege, Gesundheitsförderung, Schwangerenberatung, Sozialpsychiatrie, Suchtberatung, Behindertenberatung, Hilfen für Obdachlose usw.)

- Die Steuerung, Qualitätssicherung, Kommunikation (Gesundheitsberichterstattung, Koordinationsaufgaben, Aufsicht, Begutachtungswesen, Öffentlichkeitsarbeit usw.).

Im Bereich des Öffentlichen Gesundheitsdienstes ist aber insgesamt zu konstatieren, dass die Versorgungsforschung noch großes Entwicklungspotenzial aufweist. Wie Recherchen in der Projektdatenbank Versorgungsforschung Deutschland (Link: www.versorgungsforschung-deutschland.de) und in Literaturdatenbanken wie PubMed auf den ersten Blick ergeben, liegen relativ wenige Studien und Projekte der Versorgungsforschung zu Tätigkeitsfeldern des Öffentlichen Gesundheitsdienstes in Deutschland vor. Für den Öffentlichen Gesundheitsdienst ist die Versorgungsforschung von hoher Relevanz. Es wurde bereits im Gesundheitsbericht für Deutschland 1998 bemängelt, dass zur „Effektivität und Effizienz der Aufgabenerfüllung“ bei den Gesundheitsämtern „fast nichts bekannt ist“ [14]. Der öffentliche Gesundheitsdienst ist innerhalb der deutschen Versorgungsforschung unterrepräsentiert. Dafür sind vor allem strukturelle Gründe und die besonderen Merkmale des öffentlichen Gesundheitswesens verantwortlich. So ist z.B. das Fachgebiet Öffentliches Gesundheitswesen nicht als eigene wissenschaftliche medizinische Disziplin an einer Universität etabliert. Es gibt auch kein unabhängiges Institut oder eigenen Lehrstuhl für den Öffentlichen Gesundheitsdienst an einer medizinischen Fakultät.

\section{Versorgungsforschungsmodell für den Öffentlichen Gesundheitsdienst und das öffentliche Gesundheits- wesen \\ $\nabla$}

Die vorhandenen theoretischen Modelle und Instrumentarien der Versorgungsforschung müssen für eine verstärkte Anwendung im Öffentlichen Gesundheitsdienst und öffentlichen 


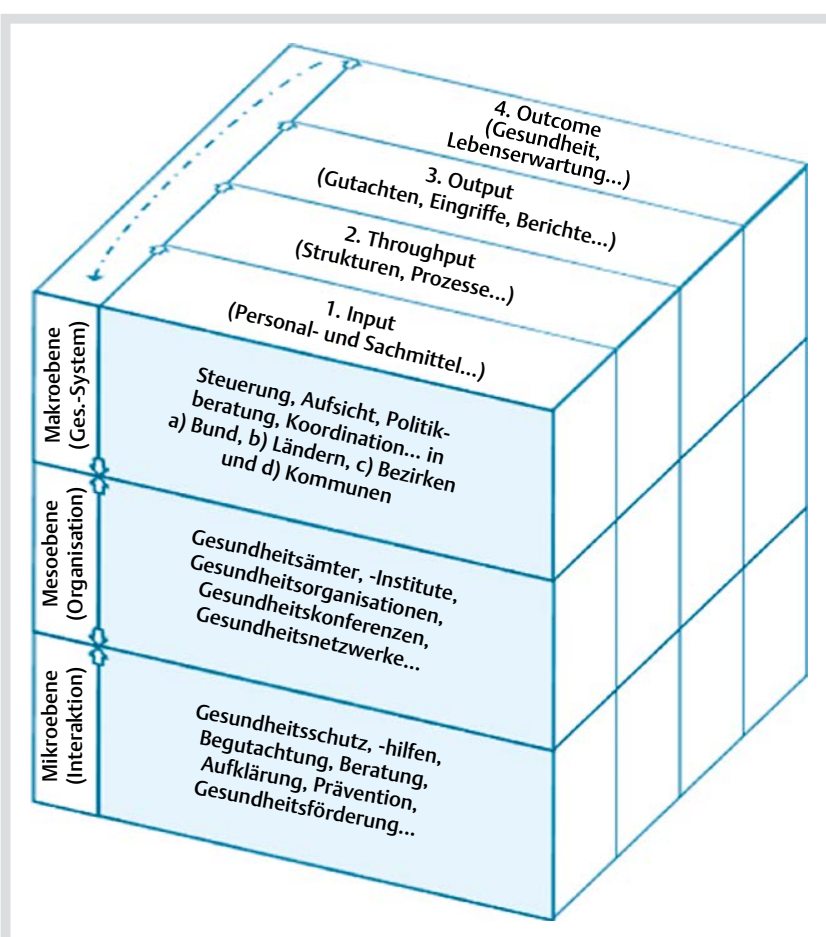

Abb. 1 Systemtheoretisches Modell für die Versorgungsforschung im Öffentlichen Gesundheitsdienst.

Quelle: Eigene Darstellung mit Rückgriff auf Modelle von Schwartz und Busse [16] und Pfaff [8].

Anmerkung: Die Detailaussagen der freien Flächen ergeben sich aus der Betrachtung der speziellen Funktionen auf den Systemebenen und ihrer Einordnung als Input/Throughput/Output/Outcome-Elemente.

Gesundheitswesen weiter spezifiziert werden. Ein theoretisches Basismodell für die Versorgungsforschung im Öffentlichen Gesundheitsdienst sollte im Anforderungsprofil eine große Kompatibilität zu dem heterogenen Aufgabenspektrum und einen hohen Anwendungsbezug aufweisen sowie den historisch gewachsenen Strukturen mit großer regionaler Varianz entsprechen. Angesichts der großen Herausforderungen im Gesundheitssystem ist die Bereitschaft und Fähigkeit der Versorgungsinstitutionen, sich auf Innovationen einzustellen, besonders wichtig [15]. Das erfordert integrationsfähige Modelle in der Versorgungsforschung, die darüber hinaus die Multidisziplinarität und -professionalität angemessen berücksichtigen.

In $\diamond$ Abb. 1 wird für diese Zwecke ein systemtheoretisches Modell der Versorgungsforschung speziell für den Öffentlichen Gesundheitsdienst als Grundlage vorgeschlagen. Es baut auf einem Modell der Gesundheitssystemforschung von Schwartz und Busse [16] und einem Modell der Versorgungsforschung von Pfaff [8] auf und bildet gesundheitssystemisch die nachfolgend beschriebene Makro-, Meso- und Mikroebene ab:

\section{a) Makroebene (Gesundheitssystem)}

Das Gesundheitssystem eines Landes bildet demnach als Ganzes die Makroebene und kann nach den politischen Ebenen weiter nach Bund, Ländern, Bezirken und Kommunen differenziert werden. Sie stellen adäquate Analyseebenen der Versorgungsforschung für den Öffentlichen Gesundheitsdienst dar, weil die Zuständigkeiten, Regionalbezüge und Rechtsgrundlagen dieser föderalen Struktur und den Gebietskörperschaften folgen. Auf der Makroebene werden wesentliche Rahmenbedingungen für die unteren Ebenen festgelegt. Hier werden die Wirkungen des Öffentlichen Gesundheitsdienstes in Bezug auf Politikberatung, Steuerungs-, Überwachungs- und Koordinationsaufgaben oder Gesundheitsberichterstattung im Zuständigkeitsbereich erforscht. Die Versorgungsforschung klärt hier z.B. die Fragen, inwieweit gesundheitspolitisch prioritäre Gesundheits-, Präventions- oder Qualitätsziele im Gesundheitssystem erreicht und Public Health-Probleme populationsbezogen gelöst werden.

\section{b) Mesoebene (Institutionen)}

Die Mesoebene fokussiert zwischen Makro- und Mikroebene die Organisationen, Settings und Netzwerke. Hier ist die organisationsbezogene Versorgungsforschung mit ihren Methoden angesiedelt [17]. Die Versorgungsforschung richtet sich auf die Einrichtungen des öffentlichen Gesundheitswesens wie die Gesundheitsämter oder Institute in den Gesundheitsressorts von Bund und Ländern. Im weiteren Sinne erfasst diese Ebene auch die Wirkungen und Qualitäten von Gesundheitsnetzwerken oder strukturierten Gesundheitskonferenzen, die häufig vom Öffentlichen Gesundheitsdienst koordiniert werden. Die Aktivitäten auf den verschiedenen Ebenen interagieren.

\section{c) Mikroebenen (Interaktionen)}

Auf der Mikroebene werden die konkreten interpersonalen Interaktionen wie in der Begutachtungs- oder Beratungssituation z.B. am Gesundheitsamt untersucht. Die Versorgungsforschung zielt auf die Qualität und Ergebnisse in der direkten Leistungserbringung für den Klienten oder Patienten. Im Öffentlichen Gesundheitsdienst stehen auf der Mikroebene besonders die Aufgaben zu Gesundheitsschutz, Diagnostik, Beratungen, Schuleingangsuntersuchungen, Screenings, Präventionsleistungen, Überwachungen und Eingriffe usw. (Output) im Vordergrund. Schätzungsweise 25-30\% der Gesamttätigkeit eines Gesundheitsamtes werden auf die Erstellung von amtsärztlichen Gutachten verwandt [18].

\section{d) „Input - Throughput - Output - Outcome“-Modell}

Zur Untersuchung des Gesundheitssystems hat sich in der Versorgungsforschung das „Input - Throughput - Output - Outcome“-Modell [8] bewährt, das sowohl auf der Mikro- als auch Meso- und Makroebene durchgängig angewandt werden kann. Zum Input zählen z.B. der Bedarf, die eingesetzten Ressourcen (Personal- und Sachmittel) oder die Inanspruchnahmen im Öffentlichen Gesundheitsdienst durch verschiedene Zielgruppen. Die Versorgungsforschung analysiert die Strukturen und Prozesse im jeweiligen Kontext (Throughput) und bestimmt die Struktur- und Prozessqualitäten. Sie sind entscheidend für die Ergebnisse der Leistungserbringung und deren Qualität (Output). An diesen Stellen kann auch die gesundheitsökonomische Evaluation ansetzen. Der Outcome meint die Gesundheitswirksamkeit der eingesetzten Methoden und Maßnahmen. Die Versorgungsforschung könnte hier bei Maßnahmen des Öffentlichen Gesundheitsdienstes untersuchen, ob z.B. einzelfall- oder populationsbezogen Zugewinne an Gesundheit, Lebensqualität und Lebenserwartung bewirkt oder Krankheitsrisiken gesenkt werden konnten. In welchem Verhältnis stehen die eingesetzten Mittel (Input) zu beobachteten gesundheitsbezogenen Veränderungen von Individuen oder Populationen (Outcome)? In der Outcome- und Evaluationsforschung besteht ein besonders großes Umsetzungsdefizit im Öffentlichen Gesundheitsdienst. Auf der Makroebene könnte sich für die Bedarfsermittlung und 
Outcome-Forschung in vielen Fällen das vorhandene System der Gesundheitsberichterstattung nutzen lassen.

Ergebnisse der Outcome- und Outputforschung können nach dem Modell auf allen Ebenen in das System iterativ rückgekoppelt werden und dabei zur Optimierung von Input, Prozessen, Strukturen oder als Grundlage für Allokationsentscheidungen beitragen. Das ist beim Öffentlichen Gesundheitsdienst für die Qualitäts- und Ergebnissicherung von besonderer Wichtigkeit, da er derzeit von Verwaltungsreformen, Personalabbau und Fachärztemangel erheblich betroffen ist.

Für die Versorgungsforschung kommt erschwerend hinzu, dass im Öffentlichen Gesundheitsdienst die geforderten randomisierten und kontrollierten Studien (RCT) [7] häufig aus rechtlichen, ethischen, gesundheitspolitischen oder historischen Gründen nicht (mehr) durchführbar sind. Die Evidenz muss dann methodisch bspw. über quasi-experimentelle oder clusterrandomisierte Studiendesigns abgesichert werden. Die starke „Alltagsorientierung“ der Versorgungsforschung als eine „grundlagen- und problemorientierte fachübergreifende Forschung" [9] kommt dagegen den spezifischen Anforderungen im Öffentlichen Gesundheitsdienst und seinen Handlungsansätzen zur Lösung von Realweltproblemen entgegen.

\section{Von „Old Public Health" zu „New Public Health“ $\nabla$}

Zur erfolgreichen Bewältigung der genannten Herausforderungen werden mehr Resultate der Begleit- und Ergebnisevaluation im Öffentlichen Gesundheitsdienst benötigt. Im Öffentlichen Gesundheitsdienst sind nicht nur die Rahmenbedingungen, sondern auch die Aufgaben selbst in Veränderung inbegriffen. Das Aufgabenprofil des Öffentlichen Gesundheitsdienstes wandelt sich nach Müller [19] unabhängig von landesspezifischen Regelungen oder kommunalen Maßnahmen. Das heutige, modernisierte Curriculum der Kursweiterbildung Öffentliches Gesundheitswesen („Amtsarztlehrgang“) sieht eine Einführung in die Gesundheitssystem- und Versorgungsforschung vor, sodass entsprechende grundlegende Kompetenzen mindestens bei den Fachärzten für Öffentliches Gesundheitswesen vorhanden sind [20]. Für die Zukunftsaufgaben im hochkomplexen Gesundheitswesen sind verstärkt Ansätze von „New Public Health“ im Öffentlichen Gesundheitsdienst erforderlich. Als „New Public Health" wird die Weiterentwicklung der wissenschaftlich angewandten Public Health-Forschung bezeichnet, die die Steuerung des Gesamtsystems und die Gesundheitsversorgung der gesamten Bevölkerung in den Mittelpunkt des Erkenntnisinteresses stellt [21]. Sie ist interdisziplinär ausgerichtet. Die Versorgungsforschung und die Gesundheitssystemforschung ergänzen die klassischen Disziplinen der Hygiene und Epidemiologie von „Old Public Health".

„Haben ÖGD und Public Health getrennt eine Zukunft?“, fragte Müller [22]. Seiner Einschätzung nach bedingen sich Public Health und Öffentlicher Gesundheitsdienst nicht nur gegenseitig, sondern die beiderseitige Zukunftsfähigkeit ist an ein gemeinsames Grundverständnis und einen konkordanten Handlungsauftrag gebunden. Public Health versteht sich in Deutschland als die „Wissenschaft und die Praxis zur Verhinderung von Krankheiten, zur Verlängerung des Lebens und zur Förderung von physischer und psychischer Gesundheit unter Berücksichtigung einer gerechten Verteilung und einer effizienten Nutzung der vorhandenen Ressourcen“ [23]. In diesem Verständnis gibt es große
Schnittmengen zu den Aufgaben des Öffentlichen Gesundheitsdienstes. Als wichtiges Tätigkeitsfeld für Public-Health-Experten werden folgerichtig die öffentlichen Verwaltungen und insbesondere der Öffentliche Gesundheitsdienst identifiziert. Die Versorgungsforschung selbst wird als eine der Kerndisziplinen von Public Health angesehen [23]. Der Öffentliche Gesundheitsdienst war in der Gründungsphase von Public Health in Deutschland zunächst nur in geringem Ausmaß beteiligt. Dies erklärt sich damit, dass der Öffentliche Gesundheitsdienst zu diesem Zeitpunkt schon nicht mehr an den deutschen Universitäten mit eigenständigen Fachvertretern präsent war. Dadurch waren einerseits Themen des Öffentlichen Gesundheitsdienstes im Wissenschaftsbereich wenig repräsentiert, andererseits fehlte ein nachhaltiger Anwendungsbezug der Forschung und Ausbildung an den neuen Public Health-Studiengängen. Die im Zuge des weiteren Ausbaus der Gesundheitswissenschaften entstandene punktuelle Kooperation mit dem Öffentlichen Gesundheitsdienst beurteilt Gostomzyk [24] „in Hinblick auf Theorie- und Methodenentwicklung im Bereich öffentliche Gesundheit für beide Beteiligten fruchtbar“. Als ein Erfolgsbeispiel von Public Health wird der Aufbau von Gesundheitsberichterstattung in Bund, Ländern und Kommunen angesehen [23].

Die neu entstandenen „Schools of Public Health“ bieten weiterhin potenziell Vernetzungsmöglichkeiten zwischen Forschung und dem Öffentlichen Gesundheitsdienst. Sie ermöglichen Kooperations- und Forschungsprojekte, aber auch Angebote für Lehre und den gestiegenen Fort- und Weiterbildungsbedarf. Als nachahmenswertes Beispiel mit Entwicklungspotenzial ist abschließend die „Pettenkofer School of Public Health München“ zu erwähnen, die als regionales Zentrum der interdisziplinären Gesundheitsforschung gegründet wurde. Hier besteht die Chance für eine stimmige, an den europaweiten „best practice“-Modellen orientierte Fortentwicklung der Facharztweiterbildung Öffentliche Gesundheit durch eine stärkere Vernetzung des Amtsarztlehrganges mit der universitären Public Health-Ausbildung. Durch eine Brückenprofessur in „Öffentliches Gesundheitswesen - Public Health Policy and Administration" wird ein wegweisender Impuls für eine (Re-)Akademisierung des Öffentlichen Gesundheitsdienstes gesetzt. Sie baut auf der langjährigen Kooperation zwischen der Ludwig-Maximilians-Universität München und der Kursweiterbildung zum Facharzt für Öffentliches Gesundheitswesen am Bayerischen Landesamt für Gesundheit und Lebensmittelsicherheit (LGL) auf. Im Rahmen der „Amtsarztweiterbildung“ können Kursteilnehmer mittlerweile erfolgreich die vertiefende akademische Ausbildung zum Master of Public Health mit Studienschwerpunkt Health Administration and Management absolvieren. Das ist ein wichtiger Schritt für eine transparente, evidenz- und wertebasierte Aufgabenwahrnehmung durch den Öffentlichen Gesundheitsdienst auf allen Ebenen. Der Namensgeber Max Josef von Pettenkofer, der erste deutsche Ordinarius für Hygiene, wusste schon 1890: „Bloßes Wissen, bloße Kenntnis von Dingen und Tatsachen allein ist noch keine Wissenschaft, erst wer etwas über Entwicklung und ursächlichen Zusammenhang der Dinge erforscht, was bisher unbekannt war, treibt Wissenschaft“" [25]. Das öffentliche Gesundheitswesen und der Öffentliche Gesundheitsdienst brauchen mehr Versorgungsforschung!

Interessenkonflikt: Die Autoren geben an, dass kein Interessenkonflikt besteht. 


\section{Literatur}

1 Johann Peter Frank-Gesellschaft, Hrsg. Gegen Armut und Krankheit. Leben und Wirken eines großen Arztes. Rodalben: Magin, 2004; 85

2 Johann PF. System einer vollständigen medicinischen Polizey. Mannheim: Schan, 1779-1819

3 Commission on Social Determinants of Health. Closing the gap in a generation: health equity through action on the social determinants of health. Final Report of the Commission on Social Determinants of Health.World Health Organization, (ed.). Genf: 2008

4 World Health Organization. Health 2020: A European policy framework and strategy for the $21^{\text {st }}$ century. WHO Regional Office for Europe, Copenhagen: 2013

5 World Health Organization. Gesundheit 2020 Rahmenkonzept und Strategie der Europäischen Region für das 21. Jahrhundert. WHO Regional Office for Europe, Copenhagen: 2013; 184

6 Schrappe M, Glaeske G, Gottwik M et al. Ständige Kongresskommission Versorgungsforschung. Konzeptionelle, methodische und strukturelle Voraussetzungen der Versorgungsforschung. Dtsch Med Wschr 2005; 130: 2918-2922

7 Gräßel E, Donath C, Hollederer A et al. Versorgungsforschung - evidenzbasiert. Ein Kurzüberblick und Implikationen. Zeitschrift „Das Gesundheitswesen“. 2014; (im Druck)

8 Pfaff $H$. Versorgungsforschung - Begriffsbestimmung, Gegenstand und Aufgaben. In: Pfaff H, Schrappe M, Lauterbach KW, Engelmann U, Halber M, Hrsg. Gesundheitsversorgung und Disease Management. Grundlagen und Anwendungen der Versorgungsforschung. Bern: Huber, 2003; 13-23

9 Bundesärztekammer. Arbeitskreis „Versorgungsforschung“ beim Wissenschaftlichen Beirat der Bundesärztekammer: Definition und Abgrenzung der Versorgungsforschung, 08. 09.2004. Berlin: 2004

10 Wildner M, Müller W, Jaeschke B et al. Der Öffentliche Gesundheitsdienst. In: Schwartz FW et al, Hrsg. Public Health: Gesundheit und Gesundheitswesen. 3. neu bearb. Aufl. München: Urban \& Fischer, 2012; 289-299

11 Bundesärztekammer. Tätigkeitsbericht 2013 der Bundesärztekammer: Ärztestatistik. Berlin: 2014

12 Bayerisches Landesamt für Gesundheit und Lebensmittelsicherheit, Hrsg. Personal im öffentlichen Gesundheitsdienst nach Berufen und Geschlecht, Bayern. Stand 03.01.2008. Indikator (K) 8.26. Im Internet: www.lgl.bayern.de Stand: 16.09.2014

13 Kuhn J, Wildner M, Zapf A. Standortbestimmung mit hoffnungsvollem Ausblick. Dtsch Arztebl 2012; 109: A 413-A 416
14 Statistisches Bundesamt, Hrsg. Gesundheitsbericht für Deutschland. Verlag Metzler-Poeschel, Stuttgart: 1998

15 Pfaff $H$, Neugebauer EAM, Glaeske G et al. Lehrbuch Versorgungsforschung: Systematik - Methodik - Anwendung. Stuttgart: Schattauer, 2011

16 Schwartz FW, Busse R. Denken in Zusammenhängen: Gesundheitssystemforschung. In: Schwartz FW et al., Hrsg. Public Health: Gesundheit und Gesundheitswesen. 3. neu bearb. Aufl. München: Urban \& Fischer, 2012; 555-582

17 Pfaff $H$, Albert US, Bornemann $R$ et al. Methoden für die organisationsbezogene Versorgungsforschung. Gesundheitswesen 2009; 71: 777-790

18 Walter K. Was ist der ÖGD, was kann der ÖGD in Deutschland? Bundesgesundheitsbl - Gesundheitsforsch - Gesundheitsschutz 2005; 48: 1095-1102

19 Müller W. Der Öffentliche Gesundheitsdienst - Fachlicher Auftrag und Bildungsangebote. Landkreis 2011; 81-83

20 Akademie für öffentliches Gesundheitswesen in Düsseldorf. Bayerisches Landesamt für Gesundheit und Lebensmittelsicherheit (LGL), Hrsg. Curriculum Kursweiterbildung „Öffentliches Gesundheitswesen“. Berichte \& Materialien Band 22: Stand Februar 2009. Düsseldorf, Erlangen: 2009

21 Hurrelmann $K$, Laaser U, Razum O. Entwicklung und Perspektiven der Gesundheitswissenschaften in Deutschland. In: Hurrelmann K, Razum O, Hrsg. Handbuch Gesundheitswissenschaften. 5. überarb. Aufl. Weinheim: Beltz Juventa, 2012; 15-51

22 Müller W. Haben ÖGD und Public Health getrennt eine Zukunft? Bundesgesundheitsbl - Gesundheitsforsch -Gesundheitsschutz 2005; 48: $1145-1152$

23 Gerlinger T, Babitsch B, Blättner B et al. Situation und Perspektiven von Public Health in Deutschland - Forschung und Lehre. Gesundheitswesen 2012; 74: 762-766

24 GostomzykJ. Der Öffentliche Gesundheitsdienst (ÖGD). In: Gostomzyk J, Hrsg. Angewandte Sozialmedizin. Handbuch für Weiterbildung und Praxis. Landsberg: Ecomed Medizin, 2006; III-2: 1-26

25 Max Josef von Pettenkofer. Rerum cognoscere causas. Ansprache in der öffentlichen Festsitzung der K. B. Akademie der Wissenschaften in München am 15. November 1890. In: Ebstein E. Deutsche Ärzte-Reden aus dem 19. Jahrhundert. Springer: 1926. Im Internet: http://gutenberg. spiegel.de/buch/6832/13. Stand: 21.05 .2014 